\title{
Adaptive Image Segmentation for Traumatic Brain Haemorrhage
}

\author{
Ahmad Yahya Dawod, Aniwat Phaphuangwittayakul

\begin{abstract}
International College of digital Innovation Chiang Mai University, 239 Nimmanahaeminda Road, Suthep, Muang, Chiang Mai 50200, Thailand
\end{abstract}

\begin{abstract}
It is challenging to establish a significant solution with computer techniques to improve the speed and efficiency of Traumatic Brain Injury (TBI) diagnosis. Several segmentation methods involving diverse precision and a degree of effort have been proposed and detailed within the related literature. Segmentation of Brain image is one of the significant clinical diagnostics implements. This paper proposes a modified (MDRLSE) calculation for haemorrhage segmentation on Computed Tomography (CT) images. The image noise that abdicates the obscured edges is utilized to portray the precise boundary of the haemorrhage region. The proposed segmentation technique achieved an accuracy rate of $97.16 \%$. The technique is implemented using an edge-based involved contour model for image segmentation, providing a simple narrowband to significantly reduce computational costs. The performance results show that it is effective for TBI image segmentation in brain images with various characteristics.
\end{abstract}

Keywords -Traumatic Brain Injury, segmentation, DRLSE, Thresholding, Morphological.

\section{Introduction}

Image segmentation is the technique of dividing a digital image into multiple sections using computer vision. The segmentation aim is to make the image

DOI: 10.18421/TEM103-61

https://doi.org/10.18421/TEM103-61

Corresponding author: Ahmad Yahya Dawod, International College of digital Innovation Chiang Mai University. Chiang Mai 50200, Thailand.

Email: ahmadyahyadawod.a@cmu.ac.th

Received: 28 May 2021.

Revised: 10 August 2021.

Accepted: 17 August 2021.

Published: 27 August 2021.

(c) BY-NC-ND (C) 2021 Ahmad Yahya Dawod \& Aniwat Phaphuangwittayakul; published by UIKTEN. This work is licensed under the Creative Commons AttributionNonCommercial-NoDerivs 4.0 License.

The article is published with Open Access at www.temjournal.com representation more understandable and easier to evaluate and turn them into anything. Image segmented items and boundaries (edges, pixel clusters, etc.) may typically be identified in imagery [1]. Therapeutic image segmentation is an essential step for most image examination assignments. For the most part, counterfeit insights and earlier information studies are combined to address the challenges of segmentation [2]. It has propelled the requirement for an application to provide a robust segmentation method, including a vast anatomic structure for sorting images and improving diagnosis. Traumatic brain injury (TBI) is caused of the brain to sudden damage such as a blow or shake to the head [3]. Normal causes involve car or motorcycle accidents, falls, and assaults. Injuries can extend from a mild concussion to severe feature damage of the brain. Severe TBI may obtain serious care and life-saving surgery. Those who survive brain damage can face lasting impacts on their natural and mental capacities [4].

TBI can involve an intracerebral haemorrhage (ICH), subdural hematoma (SDH), epidural hematoma $(\mathrm{EDH})$, cerebral wound, diffuse axonal injury (DAI), or cerebellar haemorrhage. Therapeutic imaging modalities such as CT scans are broadly utilized to assess the seriousness of the injury and the appropriate treatment during the early hours of a brain injury [5]. The brain is the foremost complex structure in the body. Distinguishing the injury locale from the normal-unaffected part of the brain is essential to ensure no wrong diagnosis is made during a critical time. A CT scan is the most effective method for detecting brain haemorrhages in humans since it operates by an x-ray tube pivoting around the patient's head to capture different images. These captured images are then analysed through a computer. The CT images allow radiologists and other medical professionals to recognize the inside structure of body mass, observe its shape, as well as estimate the thickness and surface [6]. The CT scanning method is appropriate for claustrophobic patients and those with metallic or electrical inserts in their bodies. Several techniques have been used to segment and measure haemorrhage regions in the 
brain using CT images [7]. [8] The energy function utilizes a gradient amplitude-driven end stop and the DRLSE model, however, has a number of inconveniences on DRLSE models which may segment the brain image and give crisp-border features.

First, its ending of the edge depends mostly on the slope of a brain image, so that weak targets may simply be crossed and spilled. Secondly, it requires an internal mechanism for the effective suppression of noise, rendering the segmentation particularly sensitive to noise. Thirdly, its numerical implementation requires the solution of partial differential equations and relies on. Thus, the time cost of the advance preparation is considerable. Fourthly, the segmentation results are profoundly connected with initialization. Finally, the DRLSE regularization uses the entire LSF to regard the zerolevel bend associated with the triumph or disappointment of the objective securing as its execution [9]. Brain images for the most part contain noise, inhomogeneity, and some time variation. In this manner, their exact segmentation may be an exceptionally troublesome assignment, requiring the precise, quick division of brain haemorrhage in CT images. In this study, an adjusted form of DRLSE segmentation is used which does not require reinitialization during the segmentation stage. The level is initialized, and the parameters are set according to the application. A broad dataset of CT scanning outlines involving EDH and $\mathrm{SDH}, \mathrm{ICH}$ and IVH haemorrhages is the focus of the proposed image segmentation approach as patients survive depending on their early determination, particularly ICH inside the tissue of the brain.

\section{Suggested Method}

In this paper, the seven-stage approach presented in Figure 1. is proposed: 1. CT scan images (DICOM) are converted into jpeg format; 2. Preprocessing is used to remove the skull; 3 . The threshold is obtained using median filtering; 4 . The methodology of erosion and dilation is applied; 5 . The DRLSE algorithm with LSF is utilized; 6. The region is grown by utilizing the region of interest (ROI); 7. Edge-based segmentation involving edge detection is used to provide high regional accuracy and the growing performance is assessed.

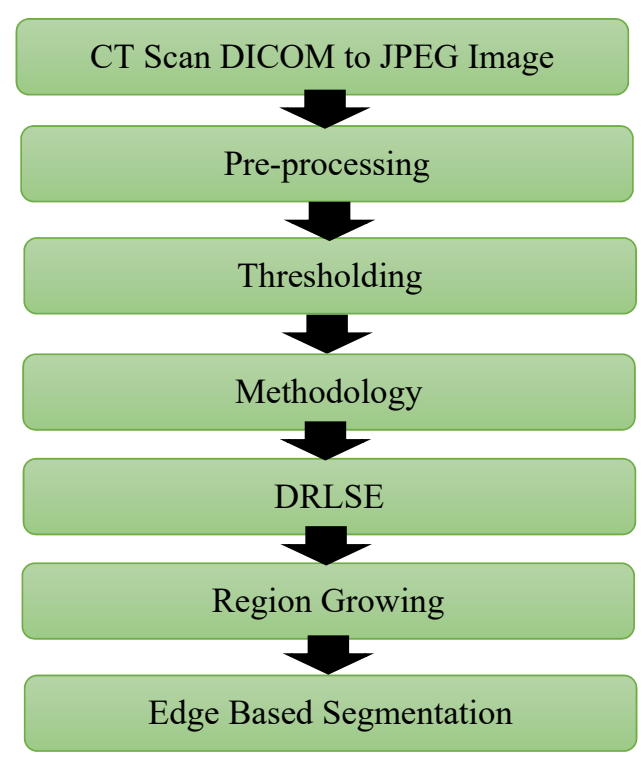

Figure 1. The proposed method is depicted as a block diagram

\subsection{Pre-Processing}

During pre-processing, the slices are selected based on the range of intracranial impedance, since both the brain and cerebral spinal liquid are encompassed by spaces that ought to be portioned. The pre-processing parameters can be balanced to suit the input images. In order to arrange the CT scan, images must be utilized as a coordinate input record for all operations concerning the change over from DICOM to JPEG format to retain the quality standard of the image pixels [10]. Such resizing is an essential step in image pre-processing. Pixel values should remain constant for each image within the dataset. They are required for different purposes, such as demonstrating the capacity and transmission of images. Whereas showing an image, the determination of the show gadgets forces imperatives on the highest estimate of the show screen. The procured image is then resized in accordance with the framework prerequisites. Within the pre-processing step, cranium expulsion and sifting of CT scans. In some recent segmentation the information was removed from critical highlights such as cranium and head holders that might be possible sources of mistakes in the filtered image. Evacuate the skull that has the most noteworthy concentration is claimed to the head. On the other hand, brain tissue is considered a challenge to brain segregation. In any case, the brain colour is dark, and the cranium colour is white by a binary image, so the skull can be expelled. The taking after strategies and scientific operations are utilized to perform the cranium evacuation. We expel the skull and the brain adaptively by taking after [11] Eq. (1).

$$
\mathrm{C}_{2}(\mathrm{x}, \mathrm{y})= \begin{cases}0 & \text { if } \quad \mathrm{P}(\mathrm{a}, \mathrm{b})<\varphi_{1} \text { or } \mathrm{P}(\mathrm{a}, \mathrm{b})>\varphi_{2} \\ \mathrm{C}_{1} & \text { Otherwise }\end{cases}
$$


Where $C_{1}$ and $C_{2}$ are, respectively, CT scan image slices and the image after the skull has been removed. $P(a, b)$ is the pixel in the a-th, row, and bth, column of the image $C_{2}, \varphi_{1}$ and $\varphi_{2}$ are defined by Eq. (2) and Eq. (3), respectively, where $\beta$ is a constant, $n_{1}$ is the image grayscale's highest value, $n_{2}$ is the median, and the image's mean is determined by Eq. (4). The removal of the skull from the original image is presented in Figure 2.

$$
\begin{aligned}
& \varphi_{1}=\beta \times \mathrm{n}_{1} \\
& \varphi_{2}=\mathrm{n}_{1}-\mathrm{n}_{2} \\
& \mathrm{n}_{2}=\text { med-mean }
\end{aligned}
$$

In this phase, there will be two problems following this removal of the skull and brain ventricles. To start with, the brain CT images include noise. Secondly, the pixels remaining after removing the skull. A mixture of noise reduction and the modification approaches for image intensity is used to resolve these challenges. The median filter is utilized by eliminating the outliers to eliminate noise. The medium filter is an excellent technique to reduce noise and keep the sharp edges of the image [11]. The intensity of the entire image is set to fill the intensity range (0-255) in order to solve the second challenge.

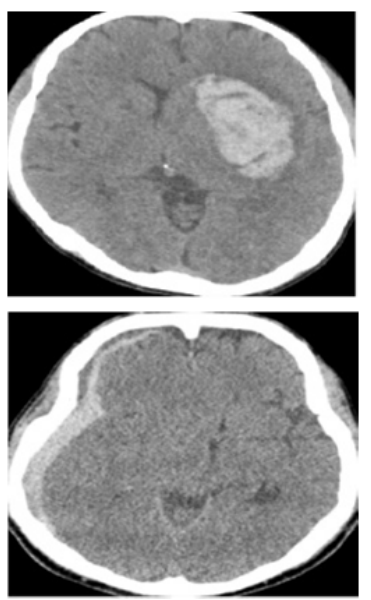

(a)
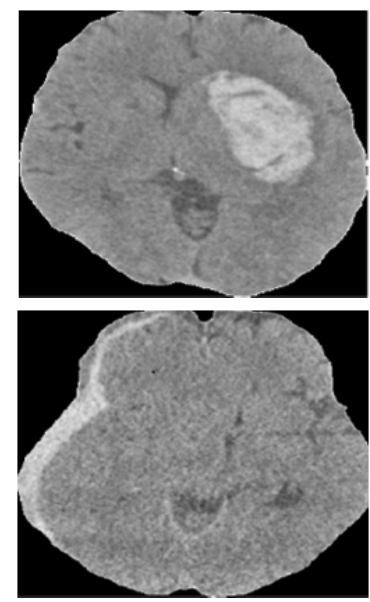

(b)
Figure 2. The brain image, (a) skull of the brain, (b) removal skull for a Subdural Hematoma

\subsection{Thresholding}

The threshold is one of the segmentation images approaches which overwhelmingly are utilized to select a fitting threshold value $T$. A moved forward threshold image segmentation algorithm based on the median filter is being utilized. This method is used to remove noise from an image or frequently used gesture rather than basically supplanting the pixel value with that of the neighbouring pixel, replacing it with the median of those pixels to connect the $2 \times 2$ square kernel filter. The median filter is chosen since it is less sensitive to strong values, making a difference without dropping the sharpness of the input image. To create a more homogeneous environment in which distortion is more self-evident, the grayscale image can be changed into a binary image to discover the haemorrhage zone by thresholding the Hounsfield units (HU) from the CT scan file to $60-85$. The results must contain all the critical data on positions and shape. The edge value $T$ obtained from the grayscale image can be classified into black (0) and white (1) [12]. The worldwide edge is utilized as in Eq. (5).

$g(a, b)= \begin{cases}1 & \text { if } f(a, b)<T \\ 0 & \text { if } \quad f(a, b) \geq T\end{cases}$

Where $f(a, b)$ is an input image, $g(a, b)$ the segmented image threshold, and $T$ the threshold value. The process of threshold segmentation is performed by estimating the threshold value $T . p_{1}$ refers to white pixels less than $T$, while $P_{2}$ refers to black pixels black more than $T$. The average intensities $n_{1}$, and $n_{2}$ of $p_{1}$ and $p_{2}$ are then applied. A new threshold value is then computed, as in Eq. (6), using different frames to indicate the threshold changes [13]. Thus, thresholding is sensitive to noise and intensity inhomogeneity defined Eq. (7):

$$
\begin{aligned}
& T_{1}=\frac{n_{1}+n_{2}}{2} \\
& \left\{\begin{array}{cc}
\text { if } \quad\left|\mathrm{T}-\mathrm{T}_{1}\right|>\Delta \mathrm{T} \\
\text { Otherwise, Stop the process }
\end{array}\right.
\end{aligned}
$$

Where $n_{1}$ and $n_{2}$ are the means of intensity $p_{1}$ and $p_{2}$ indicating the probability of white pixels as 1 and black pixels as 0 , while $T$ and $T_{1}$ are the new thresholds. The median filter connected to the segmented image is used to smooth it and remove the noise. An outstanding result can be achieved when this process is combined with region growth in segmentation as indicated in Figure 3.
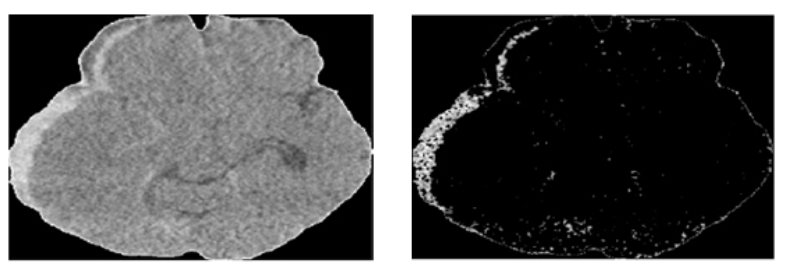

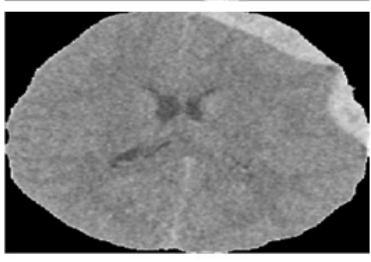

(a)

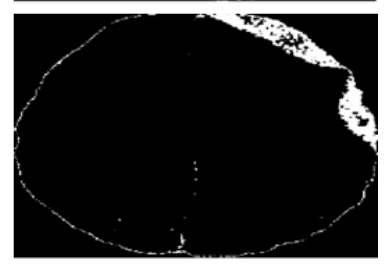

(b)
Figure 3. Thresholding steps subdural hematoma (SDH), a) original image and b) thresholding 


\subsection{Morphological Operations}

Erosion and dilation expansion are two essential morphological operations since they relate to the actualization of the parallel image [14]. Within the disintegration could be a method that employs foundation and frontal area for preparing. The binary of the output pixel has the least value (0) of all pixels within the input, while non-pixels clean the black region by removing any extra pixels which do not have a place in the white cluster on object boundaries. During the dilation process, the value of the pixel yield is the highest (1) of all pixels since a white cluster within the input pixel neighbourhood will be filled through dilation. Both can be utilized to mitigate the potential harm caused by segmentation. Dilation was accomplished following erosion of the recently extricated edge point. Erosion and dilation are characterized by Eq. (8) and Eq. (9).

$$
\begin{aligned}
& \mathrm{M} \ominus \mathrm{N}=\left\{\mathrm{Z} \backslash(\mathrm{N})_{\mathrm{Z}} \subset \mathrm{M}\right\} \\
& \mathrm{M} \oplus \mathrm{N}=\left\{\mathrm{Z} \backslash(\mathrm{N})_{\mathrm{z}} \cap \mathrm{M} \neq 0\right\}
\end{aligned}
$$

Where $M$ and $N$ are the images and organizing components, respectively. In this study, the initial operation, comprising dilation followed by erosion, is utilized to filter out the small sections within the image outside the region of suspicion, as shown by Eq. (10). The organizing component utilized in this work is level and disk-shaped, as presented in Figure 4.

$$
\mathrm{M} \odot \mathrm{N}=(\mathrm{M} \ominus \mathrm{N}) \oplus \mathrm{N}
$$

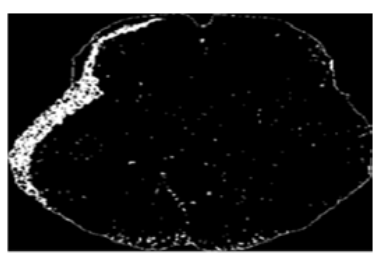

(a)

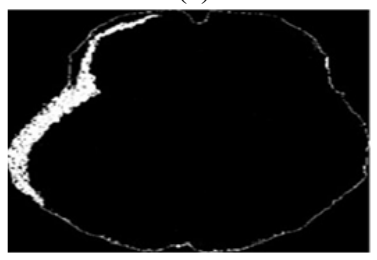

(c)

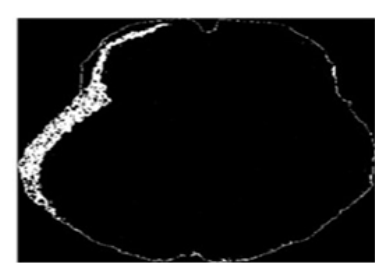

(b)

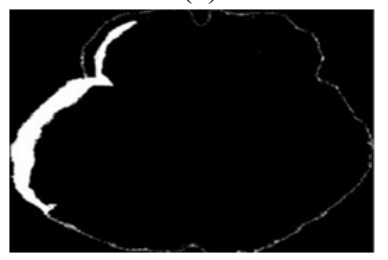

(d)
Figure 4. The morphological operations of subdural hematomas (SDH) such as $(a, b)$ Erosion and $(c, d)$ Dilation

\subsection{DRLSE Method}

In this study, segmentation was performed in two phases: shrinkage and growth. The aim of the retrenchment was to allow the zero-level outlines to enclose the region with bleeding as soon as possible [15]. It is not a matter of finding the right borders, but of dispensing with any shapes that are not haemorrhagic. The radiographic features of CT scans were used to determine the area of $50-80 \mathrm{HU}$ of blood (the blood is more hyper-intensive than in other soft tissues of the brain). The beginning of the LSF was made using the slice of the brain (axial division) emptied by the skull in the CT image. In order to ensure that dynamic contours shrunk inside the brain area without being touched on the brain borders, the initial contour, which is smallest than the brain measurement, was put in the middle of the slice. The advantage of the DRLSE is that the LSF should not be independently tagged with all parallel values for simple initialization. While the final outcome of a level strategy is the LSF zero-level set, it is crucial that the LSF is conserved in good condition, so the level set evolution is unchanging and the numerical calculation accurate. The LSF must be smooth at the smallest region of its zerolevel set amid the level set evolution. Despite the weak noise being pre-processing, such as sifting, attenuated, or eliminated solid noise caused amid the securing information or organization of different images seems to make the boundaries of the object exceptionally difficult to recognize. The first DRLSE method appropriately recognized the solid edges properly but had drawbacks in following the weak borders.

The DRLSE approach subsequently moves forward according to the application prerequisites. The zerolevel outlines were extended throughout the expansion phase to the outside. They reached the bright haemorrhage area boundaries (utilizing the edge data). This phase aimed to shield the haemorrhagic areas and meet the edges without crossing them. The DRLSE model's distance regularization, the gap between allowable and current level set distance functions will be instantly rectified. The use of active contours to segment images is a well-known technique, however the level set approach embeds active contours in a time-dependent space rather than using parametric characterization which is based on partial differential equation (PDE) functions $\phi(t, p, q)$ and complex implicit interfaces.

The evolution of active contour simplicity can then be approximated by monitoring the zero-level set. $C(t)$ function $\phi(t, p, q): \Omega \rightarrow k$ such that $\phi(t, p, q)>0$ if the point $r$ is contained by $c, \phi(t, p, q)<0$ it $(t, p, q)$ is outside $c$, and $G_{\sigma}$ if $(t, p, q)$ is ready $c$. The energy performance of the DRLSE model is improved with the level set representation.

It's worth noting that using the time variable results in the higher-dimensional level set function $\phi$, which requires a little more work but has a lot of benefits. The contour $C$, for example, can be easily calculated 
by testing the values of the level set function $\phi$, which naturally accommodates the implicit contour's $C$ topological changes. The numerical level collection determines the evolution $\phi$ as in Eq. (11).

$$
\left\{\begin{array}{c}
\frac{\partial \phi}{\partial t}+F / \nabla \phi=0 \\
\phi(0, p, q)=\phi_{0}(p, q)
\end{array}\right\}
$$

Where $\mu$ and $\lambda$ are positive control constants, $\alpha$ is a control constant in the real number range, $\phi$ is the LSF. The length regularization term $L_{r}(\phi)$ and the region regularization term $A_{r}(\phi)$. The DRLSE model's external energy concept is made up of these two terms $L_{r}(\phi)$. The function of the duration regularization term $A_{r}(\phi)$, in contrast to Eq. (12), to increase the rate of evolution in the LSF [15].

$$
r=\frac{1}{1+\left|\nabla\left(G_{\sigma} * I\right)\right|^{2}}
$$

Where does the smoothing Gaussian kernel $G_{\sigma}$ and the $G_{\sigma} * I$ standard deviation $\sigma$ represent the convergence of the image $I, r$ is near zero in variational boundaries, $\nabla$ denotes the gradient operator, several of the highest restrictions start within the unique DRLSE approach such as the calculation time at the point affecting the first joining area and the latest number of iterative phases in the request for joining. LSFs on high contrast images require little emphasis to reach a uniform shape, while in low contrast images' extra accents may be necessary. Consequently, a feasible strategy to stop the evolution of the LSF must be studied to offer high intensity inside the ROI, as illustrated in Figure 5 .

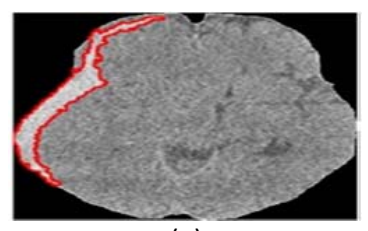

(a)

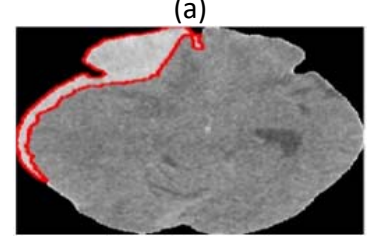

(c)

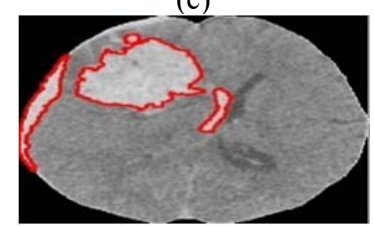

(e)

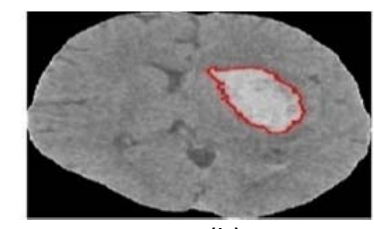

(b)

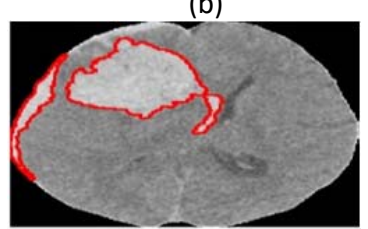

(d)

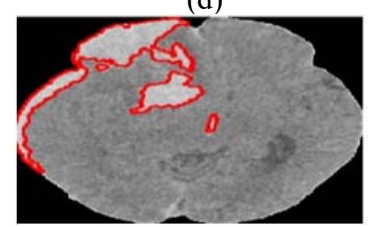

(f)
Figure 5. Growth of the LSF to the regions of interest, a) Subdural Hematoma (SDH), b) Epidural Hematoma (EDH). c), Two features Hematoma, d) Three features Hematoma, e) four features Hematoma, f) five features Hematoma

If $r$ is supposed to be 1, DRLSE may simplify the second phrase as far as $\operatorname{div}\left(\frac{\nabla \phi}{|\nabla \phi|}\right)$ the original DRLSE model values the mean of curvature $k$ and $v$ is the customizable balloon force. If DRLSE's second term value is still fixed, regularization and smoothing techniques on the curve length may lose some visual features if the image contains noise disruption. This approach relies on a penalty word in the vitality definition that penalizes the LSF's divergence from a signed distance function. The DRLSE is written as Eq. (13).

$$
\frac{\partial \phi}{\partial t}=r|\nabla \phi|\left(\operatorname{div}\left(\frac{\nabla \phi}{|\nabla \phi|}\right)+v\right)
$$

\subsection{Region Growing}

The region growing technique is a prominent segmentation approach, beginning with a seed pixel and growing by including the neighboring pixels based on a threshold value. At the point when the growth of a region stops, another seed pixel not yet belonging to a region is selected, and the procedure is repeated [16]. The growth is halted when all pixels belong to a region. The system of the region is separated from an image into comparative homogenous areas of associated pixels through the utilization of homogeneity or similar criteria among up-and-comer sets of pixels. Every pixel in a region is comparable to certain qualities or processed property, such as colour, intensity, and texture. To begin region growing, a seed must be chosen according to specific criteria and should be selected automatically. It depends upon the intensity value of neighbouring pixels as well as threshold value if the intensity value of neighbouring essential-have minimum distance with its neighbour pixels is the same and it lies in the given threshold value our region will start to grow and the mean of the region utilized as a measure of similarity. It checks those recently visited pixels if a pixel is as of now grown i.e., part of our region, it won't be indicated again on the issue on the off chance that it comes as a neighboring pixel be disengaged. This will reduce computational overhead. Be that as it may, when the region starts to grow it needs a stopping gradient to confine the growth of the area to the ROI. The pixel with the base contrast estimated is apportioned to a specific region. The pixel with the base contrast estimated is apportioned to a specific region. This procedure stops when the region is unable to expand more from the specific seed. The region growing outputs are demonstrated in Figure 6. 


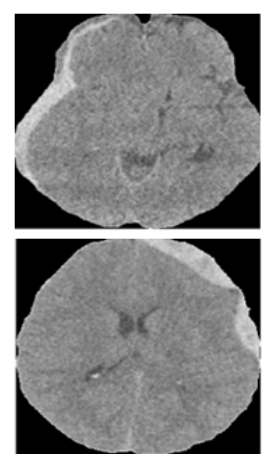

(a)

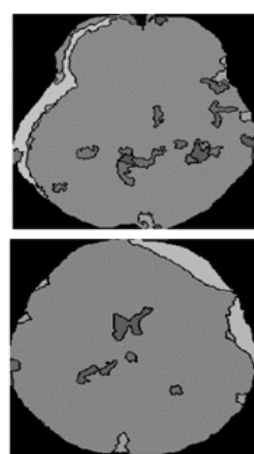

(b)

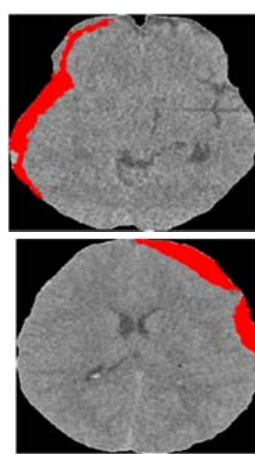

(c)
Figure 6. The haemorrhage for region Merging, a) Original Image, b) Thresholding, c) Region Merging

\subsection{Edge-Based Segmentation}

Pixels are categorized as edge or non-edge in edgebased segmentation based on the filter applied to the image. Any pixels not divided by an edge are allocated to the same category. There are two fundamental edge-based segmentation techniques: the black histogram (threshold value), and the inclination-based approach. The area limit or an item, and that article's number of intrigues is equivalent to the number of boundaries in an image [17]. When there is a sudden change in force close to the edge, and there is little image commotion, then the anglebased technique functions admirably. It is determined by including convolving slope or first and secondrequest subsidiaries. The Canny edge recognition calculation is used in computer vision and the image preparing method for the evacuation of clamour and finding the force slope of the edge-based segmentation approach. The shrewd edge calculation chooses the $2 \times 2$ neighbor region for the count of the image inclination and gains the bearing of the size and gradient direction. Edge-based segmentation is utilized to identify the limits between two unique areas. The algorithm first identifies the edges of the image by applying the subordinate administrator. Secondly, it discovers the pixel value at the edges. Thirdly, it retains all edges with a pixel value greater than threshold value $T$. Fourthly, it detects the location of the edge, checking the neighbouring pixel value. Finally, stages three and four are repeated with different estimations of the edge until the closed boundaries are identified. The segmentation of an image is presented in Figure 7.

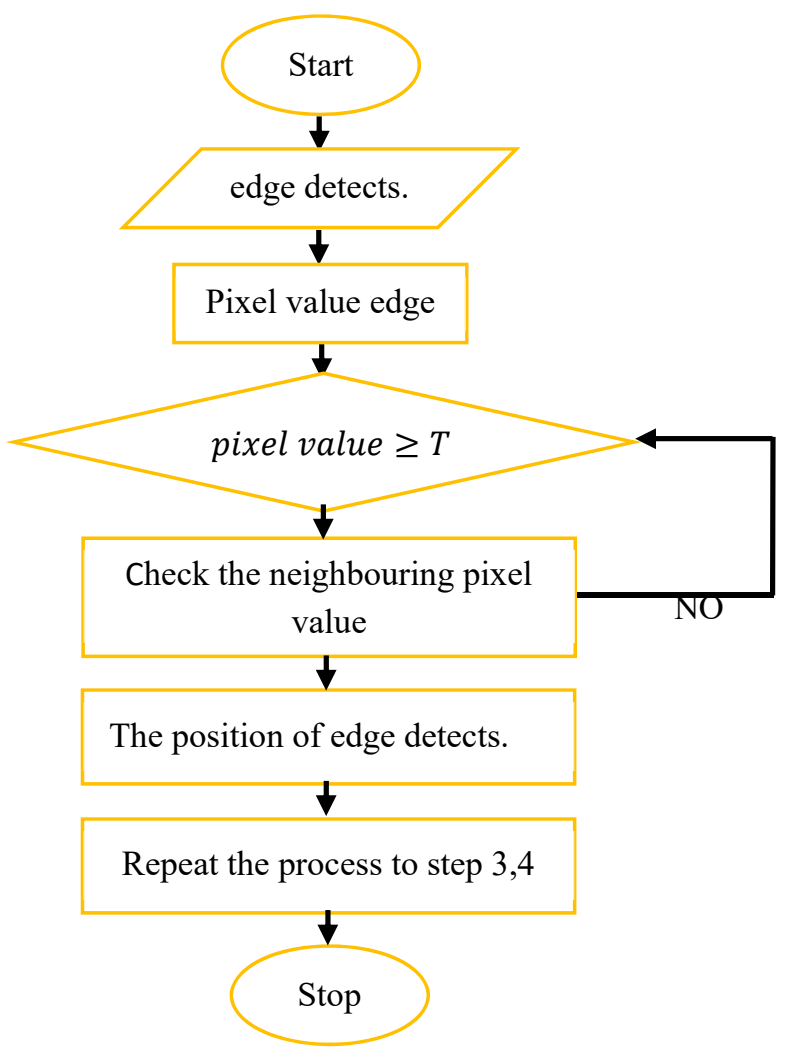

Figure 7. Flowchart for the edge detection algorithm

The following critical stage provides a path from the image dataset to the system directory. It can be implemented using an Anaconda framework to allocate datasets from computer memory through the Python OS built-in operating system. Segmentation aims to cluster pixels into image regions and identify an ROI, i.e., an injury or other abnormality. The segmented portion of the pixels is compared to the normal brain image using the Spyder notebook to locate abnormal parts of the brain. The next step is to detect the edges to classify the injured portion of the segmented image, as shown in Figure 8. (a)

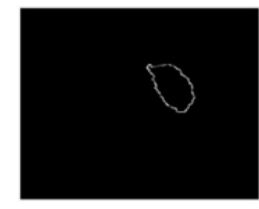

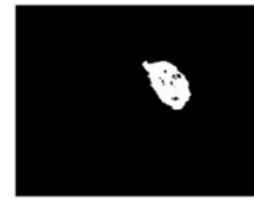

(b)

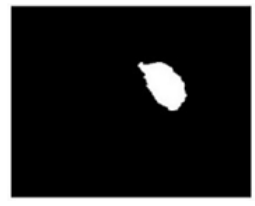

(c)
Figure 8. Different steps for Edge Detection result for Segmentation. a) Edge Detection, b) Counter Edge, Haemorrhagic Regions 


\section{Datasets}

The study dataset comprises of CT scans of patients suffering from both intra-axial and extraaxial injuries using Axial slices of digital imaging and communications in medicine (DICOM). The proposed approach investigates brain haemorrhages to evaluate a set of distinctive image classifiers comprising more than 6,520 labelled slices ( $\mathrm{ICH}$, $\mathrm{EDH}, \mathrm{SDH}, \mathrm{MLS}, \mathrm{IVH}$, and normal). The brain haemorrhage image slices were obtained from specialists at Maharaj Nakorn Chiang Mai Hospital, Thailand using 20,000 brain haemorrhage images. The thickness of the slices inside a volume was steady in most datasets, while 700 patients' datasets showed variations in the thickness of slices between male and female patients aged between 19 and 70 . The slice thickness chosen varied from 1.0 to $1.5 \mathrm{~mm}$ for each of the 100-350 slices. Haemorrhage boundaries are usually irregular and indistinct with discontinuities, presenting extraordinary challenges, particularly against conventional edge-based approaches. Furthermore, brain haemorrhage CT data obtained from clinical scans or engineered datasets are intrinsically problematic.

Table 1. The number of original patients haemorrhage features

\begin{tabular}{|c|c|c|c|c|}
\hline Normal & (SAH) & (EDH) & (ICH) & (SDH) \\
\hline 55 & 156 & 135 & 170 & 184 \\
\hline
\end{tabular}

CT scans and conventional devices utilized for procurement can drastically change scan-to-scan radiants, escalating predispositions, and other contrasts for each different slice of the image within the dataset. Moreover, the prerequisite for a few shapes to successfully portion haemorrhage subregions significantly mitigates this difficulty. These constriction measurements are mapped to a selfassertive scale of -1000 (air) to +1000 (bone) in CT scanning, as indicated in Figure 9. The constriction coefficient determines the intensity of the tissue or bone. The higher value presents the brighter region inside the CT scan. In a CT scan, the intrigue tissue can be detected by windowing. The Hounsfield unit (HU) values of the skull bones and calcified regions are greater than those of other parts of the brain and haemorrhage blood; additionally, haemorrhage blood has higher HU values than cerebral spinal fluid (CSF) and soft tissues. The HUs can be changed to greyscale by setting the window width to 151 and the window level to 114 . The Hounsfield values are within the run of 50-95 to coincide with those of brain haemorrhages. The mapping function is expressed in Eq. (14).

$$
\mathrm{CT}_{\text {Number }}=\frac{\mu-\mu_{\text {water }}}{\mu_{\text {water }}} \times 1000
$$

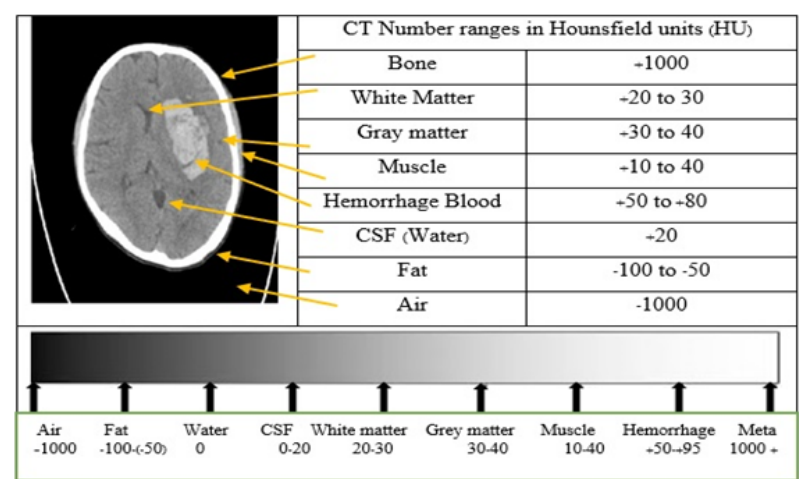

Figure 9. Computed Tomography in imaging and angiography

\section{Result and Discussion}

To build its key external driving forces, the edgebased segmentation method typically relies on the image's gradient features. In the event of a sufficiently visible target gradient amplitude and limited background noise interference, this approach can give high-quality segmentation results. In regionbased approaches, statistical information from the inner and outer areas divided by active contours is often employed to generate external driving forces, with common statistical artifacts like strength, colour, and texture changing domain properties. When compared to edge-based approaches, such approaches offer significant advantages of edge capture, reduction of background interferences, noise resilience, and other factors. Due to their distinct colour strength, these areas can be easily identified by naked eyes. The slices are defined in detail on a black background with white text. White is the colour of the skull. The ventricles are dark grey, and the grey matter is light grey. A haemorrhage is normally darker in colour than the skull and lighter in colour than the grey matter.

The proposed methodology has been evaluated on a dataset of 700 patients $(250$ image slices with a thickness of $1 \mathrm{~mm}$ ). The internal design and area of haemorrhagic injuries are significant because the presence of an interior structure aids the understanding of hematomas or separate idiopathic. In contrast to $(\mathrm{ICH}),(\mathrm{SDH})$ and $(\mathrm{EDH})$ are caused by pathological conditions. Brain haemorrhage segmentation is performed in several stages, each involving shapes of the haemorrhage region. The methodology consists of seven steps. Firstly, the skull shape is removed from the brain image, the median filtering is used with thresholding to remove the noise from the brain. Morphology is then used on the hemorrhage region inside the brain to remove the noise from the brain and identify the haemorrhage shape. The DRLSE algorithm subsequently identifies the boundary of the haemorrhage region, before growing it for segmentation. Applying this 
methodology to images with obvious edges be able to generally achieve a great segmentation performance. An image segmentation framework was created for this study based on the DRLSE model. A variable contour length regularization model for the initial DRLSE prototype was designed, which can change depending on the image region's apparent properties. The corresponding to the smooth zone in the absence of the target or the undulating region in the presence of the target to solid regularization in the area adjacent to the haemorrhage boundary to minimize the likelihood of evolution. As an adaptive variable in the initial DRLSE model, its value adjustments as the regional appropriate require changes. The performance of the edge detection feature in the innovative DRLSE model is improved without noise or anti-noise. The new edge detection performance will still maintain an applicable evolution speed while not locating noise. A regularisation term was also added for dealing specifically with the zero-level edge pixels. The expanded compound regularisation method further improves the noise destruction ability and flexibility toward the weak edges of the proposed template.

The adaptive regularization provides a significant expansion of the regularization procedure for the basic DRLSE models, which increases the noise ability and the flexibility of the border in this methodology. The edge-based segmentation methodology has achieved unrivalled performance for data segmentation of high-quality images; however, the noise is large, or the gradient of the lens is not clear, the precision of the segmentation is not much affected. The performance evaluation of the algorithms using precision and recall measures was conducted using quantitative analysis. The segmentation results are quantitatively estimated. A haemorrhage region identified by a qualified radiologist from Maharaj Nakorn Chiang Mai Hospital was used as the dataset. Three quantitative measures, namely precision, recall, and overlap metrics, were used to evaluate the segmentation results. In order to evaluate and recall the suggested model's effectiveness, we employ Dice Similarity Coefficient (DSC), Union Intersection (IoU) and Hausdorff distance (HD), and Location Accuracy (LA). The Dice similarity coefficient (DSC) is the most often used statistic to compare manual versus automated segmentation, often known as the Dice rankings. The predictor formula is quantitative:

$$
D S C=\frac{I C H_{P} \cap I C H_{G}}{\left|I C H_{P}\right|+\left|I C H_{G}\right|}=\frac{2 \times T P}{2 \times T P+F N}
$$

True positive (TP) signifies the pixel has been successfully forecast by the model. Incorrectly, a pixel that the model assesses as positive is the false positive (FP). The term false-negative (FN) refers to when the model incorrectly classifies a positive pixel as a negative one. Dice values range from 0 to 1 (where 0 indicates no overlapping pixels and 1 indicates total segmentation). Union and Intersection (IoU): Intersection over Union is a metric for determining how accurate an object detector is on a given dataset. $I o U$ can be applied to test any process that produces expected bounding boxes as an output. The segmentation measuring statistic is the Jaccard Index Intersection over Union (IoU). It may be understood as a similarity metric between few sets [18]. The percentage of the region of connection between the ground truth $\left(I C H_{G}\right)$ and predicted segmentations $\left(\mathrm{ICH}_{\mathrm{P}}\right)$ to the region of union between the ground truth and predicted segmentation. The following can be described for a similarity metric among two sets of intersection by union:

$$
I o U=\frac{I C H_{P} \cap I C H_{G}}{I C H_{P} \cup I C H_{G}}=\frac{T P}{T P+F P+F N}
$$

$I o U=1$ if the forecast is correct, and $I o U=0$ if it loses. An IoU meaning between those two is determined by the degree of overlap. Dice and $I o U$ both have a range of 0 to 1 but $I o U$ is typically smaller than Dice. This is due to the following partnership between them as Eq. (17):

$$
\frac{\text { Dice }}{I o U}=2-\frac{2 T P}{2 T P+F P+F N} \geq 1
$$

$I o U$ offers a broader range of statistical evaluations than DSC in the case of the weak segmentation in particular [19]. The Dice coefficient, on the other hand, changed linearly as the number of overlapping regions improved, while the IoU changed nonlinearly. As a result, DSC is a more intuitive way to assess procedure development. A value close to 1 in $D S C$ and $I o U$ represents the most accurate segmentation.

The Hausdorff distance $H D$ is a common performance metric for calculating the distance between two sets of points [20]. It is used in medical image segmentation to equate ground truth images with segmentations in order to rate them. $H D$ is a measurement of a set's maximum length distance from the nearest point in another set. It implies that the segmentation is good when the value is near to 0 . The Euclidean distance is defined by $d($.

$$
H D=\max \left\{d \left(I C H_{P}, I C H_{G}, d\left(I C H_{G}, I C H_{P}\right\}\right.\right.
$$

Location Accuracy (LA): The accuracy of detecting lesions is measured by location accuracy (LA). One of the most essential purposes of the automated lesion diagnostic approach is to find the lesion field, which may give a rapid view to lead the region to be thoroughly differentiated. In this 
example, it is stated whether the output can find the proper value $\alpha$. In the image, there is a lesion area for haemorrhage. $N$ is image number in the dataset. It can be summed up as follows [21]:

$$
\begin{aligned}
& L A=\sum_{i=1}^{N} \alpha / N \\
& \alpha=\left\{\begin{array}{ll}
1, \text { if } & I C H_{P} \cap I C H_{G} \neq 0 \\
0, \text { if } & I C H_{P} \cap I C H_{G}=0
\end{array}\right\}
\end{aligned}
$$

Several performance measures were developed to compare the anticipated segmented lesions with the data set. Among the criteria utilized were the true positive rates (TPR), sometimes called sensitivity and/or remembrance, the true negative rate (TNR), also known as specimen or precise, positive predictive value (PPV), precision (ACC). True positive pixels (TP) are positive predicted and defined as positive by the evaluated settings, genuine negatives (TN) are negative pixels, and false positive (FP) are predicted to be negative by the evaluated configuration. The pixels are negative, respectively, predicted to be negative and identified by the evaluated settings. The following are specified for pixels labelled as Positive and False Negation (FN) and pixels anticipated as Positive but discovered to be Negative [22]:

TPR: Identified as sensitivity or a reminder, this is a measure compared to the number of True Positive Cases:

$$
T P R=\frac{T P}{T P+F N} \times 100 \%
$$

TNR: A method which compares the rate of genuine negative to the number of real negative occurrences as given in Eq. (22) is known as accuracy or specificities.

$$
T N R=\frac{T N}{T N+F P} \times 100 \%
$$

PPV: Identified as precision, is a metric that compares the proportion of true positives to all expected positives, as seen in Eq. (23).

$$
p p v=\frac{T P}{T P+F P} \times 100 \%
$$

ACC: This is a statistical bias test that indicates how similar the forecasts are to the True values.

$$
A C C=\frac{T P+T N}{T P+T N+F P+F N} \times 100 \%
$$

F1: The F1- score is used to determine how close the predicted injury boundary is to the ground truth frontier as shown in Eq. (25) and is defined by the injury mean of accuracy and repetition values with a distance error tolerance to determine if a point on the predicted boundary corresponds to the ground truth boundary.

$$
F_{1}=\frac{2 T P}{2 T P+F P+F N} \times 100 \%
$$

For an accurate diagnosis of brain abnormality in patients, a proper segmentation method is required for CT scan images to improve diagnosis and treatment. Data is currently provided by numerous images from different brain slices for diagnosis, planning, and treatment purposes. Evaluation of image segmentation performance is challenging for the medical image analysis system. Accuracy is crucial to the proposed segmentation technique since it relates to the degree to which the overcome agrees with the dataset. For the TP, TN, FP, and FN, the recall is considered to be the same as TPR, and precision the same as TNR in Table 2.

Table 2. Slices between Umber 30 - 80 of each patient

\begin{tabular}{ccccc}
\hline Features & TP & TN & FP & FN \\
\hline EDH & 374 & 66 & 7 & 11 \\
ICH & 113 & 70 & 5 & 3 \\
SDH & 542 & 112 & 10 & 6 \\
\hline
\end{tabular}

Table 3., begins from slice number 30 to slice number 80 , using different statistical parameters while Table 4 . starts from slice number 40 to slice number 80 , showing different shapes to evaluate the three features employed for testing each patient, namely $E D H, I C H$, and $S D H$ to analyse the relevant recall, precision, and accuracy.

Table 3. Beginning from slices between Umber $30-80$ of each patient

\begin{tabular}{cccccc}
\hline Precision & Recall & F1 & Accuracy & PPV & NPV \\
\hline 90.41 & 97.14 & 93.65 & 96.06 & 98.16 & 85.71 \\
93.33 & 97.41 & 94.95 & 95.81 & 97.41 & 95.89 \\
91.18 & 98.91 & 94.87 & 97.61 & 98.18 & 94.49 \\
\hline
\end{tabular}

This study attempts to evaluate the performance of recent research works on brain abnormality detection and segmentation. Through analysis of the literature, the automation of brain abnormality detection and segmentation from CT images was found to be one of the most active research areas, with an enormous amount of research conducted in recent years. The overlap measurement, however, depends on the object's size and form complexity. As indicated in Table 4., the strategy provided in this study is compared with four further investigations. The emphasis of this comparison is the accuracy and performance of the three characteristics. 
Table 4. Slices between Umber $40-80$ of each patient

\begin{tabular}{ccccc}
\hline Features & TP & TN & FP & FN \\
\hline EDH & 310 & 40 & 8 & 7 \\
ICH & 482 & 61 & 13 & 11 \\
SDH & 120 & 37 & 7 & 5 \\
\hline
\end{tabular}

The form of the pixel and its close-fitting properties identified the skull free subdural bleeding. Haemorrhages observed on the left lobe of the brain are a cerebral and intracranial haemorrhage, which have no link to the section of the skull. The skull premovement method is utilized to determine a brain haemorrhage, however the skull removal is not utilized by other forms of brain bleeding. Thus, the measurement and location of irregularity depends on different types of bleeding. Precision metrics used to evaluate various writers' performance are as illustrated in Table 5.

Table 5. Beginning from slices between Umber 40 - 80 of each patient

\begin{tabular}{cccccc}
\hline Precision & Recall & F1 & Accuracy & PPV & NPV \\
\hline 83.33 & 97.77 & 89.45 & 97.89 & 97.48 & 85.10 \\
82.43 & 97.61 & 88.87 & 97.76 & 97.37 & 84.72 \\
88.09 & 96.33 & 91.82 & 97.71 & 94.48 & 88.11 \\
\hline
\end{tabular}

We compare our method with another research papers as shows in Table 6 . by using three features of haemorrhage (EDH, ICH, SDH) with the average of them.

Table 6. Retrieval accuracy of the haemorrhage by using different papers

\begin{tabular}{ccccc}
\hline Method & EDH & ICH & SDH & Average \\
\hline$[23]$ & 96.15 & 95.96 & 94.87 & 95.66 \\
{$[24]$} & 96.00 & 96.00 & 92.00 & 94.66 \\
{$[25]$} & 96.00 & 93.34 & 95.00 & 94.78 \\
{$[26]$} & 97.60 & 99.20 & 97.00 & 97.93 \\
{$[27]$} & 86.3 & - & 84.3 & 85.3 \\
{$[28]$} & 72.0 & 87.0 & 84.0 & 81 \\
{$[29]$} & 62.3 & 82.2 & 54.1 & 66.2 \\
{$[30]$} & 48.9 & 88.4 & 71.8 & 69.7 \\
{$[31]$} & 72.7 & 96.6 & 89.1 & 86.1 \\
Our Method & 97.06 & 99.81 & 96.61 & 97.16 \\
\hline
\end{tabular}

The case in Figure 10. (a) edge detection image of haemorrhage, while Figure 10. (b) has image segmentation with high accuracy. Table 7 . shows the results, which are dependent on DSC, IoU, HD, ACC . We were unable to segment the brain lesion using various techniques, so we only compared our findings with the two datasets described above. We can see that our method gives the high DSC and IoU metric value and low $H D$ value. Our method outperforms the existing methods with the highest accuracy.

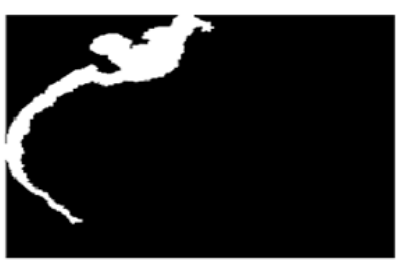

(a)

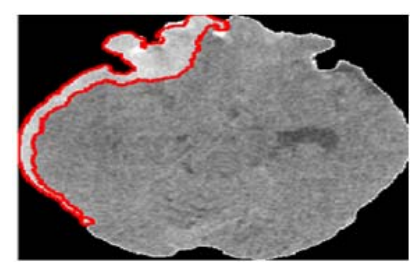

(b)
Figure 10. The performance of segmentation with brain haemorrhage, (a) the segmentation of an image, (b) output contours

Table 7. Comparison of three datasets to evaluation metrics obtained for the images

\begin{tabular}{lllll}
\hline Method & IoU & DSC & HD & ACC \\
\hline Unet++ [31] & 0.64 & 0.17 & 0.53 & 70.25 \\
Unet [25] & 0.24 & 0.24 & 0.38 & 65.29 \\
Our Method & 0.71 & 0.42 & 0.68 & 90.38 \\
\hline
\end{tabular}

The subdural haemorrhage value is presented in the table, including the proposed methods are the number of pixels (NB) and relative area error (RE), with different slices determining the relative area error giving the best results shown in Table 8 .

Table 8. The area error and number pixel region for haemorrhage

\begin{tabular}{llll}
\hline Slices & NB & RE & Image haemorrhage shape \\
110 & 11869 & 2.03 \\
111 & 11977 & 1.73 \\
112 & 11651 & 2.47 \\
113 & 11603 & 1.90
\end{tabular}

\section{Conclusion}

The proposed segmentation method has successfully provided accurate measurements for slice thickness, haemorrhage region, and the localization of lesions from CT scans. An automated edge-based segmentation algorithm was developed in this study, applying a modified version of the DRLSE method. Several problems were overcome by using the DRLSE method, with the model being able to achieve high segmentation performance in terms of accuracy, speed, and robustness. All these improvements have led to the development of a robust algorithm for medical image segmentation. A perfect set of shapes in the haemorrhage regions can be detected, even the smallest and the most uncertain. Automated systems for evaluating and categorizing medical images are attracting a lot of interest, and the approaches provided will help to improve them. The 
results obtained are encouraging with greater accuracy in the three features (SDH), (EDH), and intracerebral haemorrhage are all examples of difficulties (ICH). Different sorts of medical imaging have been used to evaluate performance. This study aims to increase the accuracy of a physician's diagnosis, saving the lives of more people as a result. In future research, the continued development of high-performance segmentation models is proposed by applying more features of a brain haemorrhage to clustering, for more the challenges of big data for CT scans. And also, we involve the AI field by using a deep learning algorithm for the classification.

\section{Acknowledgments}

The researchers wish to thank the international college of digital innovation Chiang Mai University and supported by the Faculty of Medicine Research Fund, grant no. 143-2562 to help our group to collect the datasets of haemorrhage CT scan images.

\section{References}

[1]. He, W., Wang, L. S., Li, H. Z., Cheng, L. G., Zhang, M., \& Wladyka, C. G. (2013). Intraoperative contrastenhanced ultrasound in traumatic brain surgery. Clinical imaging, 37(6), 983-988.

DOI: 10.1016/j.clinimag.2013.08.001

[2]. Chen, S., Feng, H., Sherchan, P., Klebe, D., Zhao, G., Sun, X., ... \& Zhang, J. H. (2014). Controversies and evolving new mechanisms in subarachnoid hemorrhage. Progress in neurobiology, 115, 64-91. DOI: 10.1016/j.pneurobio.2013.09.002

[3]. Cristofori, I., \& Levin, H. S. (2015). Traumatic brain injury and cognition. Handbook of clinical neurology, 128, 579-611.

DOI: 10.1016/B978-0-444-63521-1.00037-6

[4]. Ledig, C., Heckemann, R. A., Hammers, A., Lopez, J. C., Newcombe, V. F., Makropoulos, A., ... \& Rueckert, D. (2015). Robust whole-brain segmentation: application to traumatic brain injury. Medical image analysis, 21(1), 40-58.

DOI: 10.1016/j.media.2014.12.003

[5]. Zacks, J. M., Kurby, C. A., Landazabal, C. S., Krueger, F., \& Grafman, J. (2016). Effects of penetrating traumatic brain injury on event segmentation and memory. Cortex, 74, 233-246. DOI: $10.1016 /$ j.cortex.2015.11.002

[6]. Kamnitsas, K., Ledig, C., Newcombe, V. F., Simpson, J. P., Kane, A. D., Menon, D. K., ... \& Glocker, B. (2017). Efficient multi-scale 3D CNN with fully connected CRF for accurate brain lesion segmentation. Medical image analysis, 36, 61-78.

DOI: 10.1016/j.media.2016.10.004

[7]. Soltaninejad, M., Lambrou, T., Qureshi, A., Allinson, N. M., \& Ye, X. (2014, July). A Hybrid Method for Haemorrhage Segmentation in Trauma Brain CT. In MIUA (pp. 99-104).

DOI: https://doi.org/10.3174/ajnr.A5742
[8]. Havaei, M., Davy, A., Warde-Farley, D., Biard, A., Courville, A., Bengio, Y., ... \& Larochelle, H. (2017). Brain tumor segmentation with deep neural networks. Medical image analysis, 35, 18-31.

DOI: 10.1016/j.media.2016.05.004

[9]. Chen, H., Dou, Q., Yu, L., Qin, J., \& Heng, P. A. (2018). VoxResNet: Deep voxelwise residual networks for brain segmentation from 3D MR images. NeuroImage, 170, 446-455.

DOI: 10.1016/j.neuroimage.2017.04.041

[10]. Chaudhary, N., Pandey, A. S., Gemmete, J. J., Hua, Y., Huang, Y., Gu, Y., \& Xi, G. (2015). Diffusion tensor imaging in hemorrhagic stroke. Experimental neurology, 272, 88-96.

DOI: 10.1016/j.expneurol.2015.05.011

[11]. Shahangian, B., \& Pourghassem, H. (2016). Automatic brain hemorrhage segmentation and classification algorithm based on weighted grayscale histogram feature in a hierarchical classification structure. Biocybernetics and Biomedical Engineering, 36(1), 217-232.

DOI: $10.1016 /$ j.bbe.2015.12.001

[12]. Gautam, A., Raman, B., \& Raghuvanshi, S. (2018). A hybrid approach for the delineation of brain lesion from CT images. Biocybernetics and Biomedical Engineering, 38(3), 504-518.

DOI: $10.1016 /$ j.bbe.2018.04.003

[13]. Dhal, K. G., Das, A., Ray, S., Gálvez, J., \& Das, S. (2020). Nature-inspired optimization algorithms and their application in multi-thresholding image segmentation. Archives of Computational Methods in Engineering, 27(3), 855-888.

DOI: $10.1007 / \mathrm{s} 11831-019-09334-y$

[14]. Aslam, A., Khan, E., \& Beg, M. S. (2015). Improved edge detection algorithm for brain tumor segmentation. Procedia Computer Science, 58, 430437. DOI: 10.1016/j.procs.2015.08.057

[15]. Jayaraman, T., Reddy, S., Mahadevappa, M., Sadhu, A., \& Dutta, P. K. (2020). Modified distance regularized level set evolution for brain ventricles segmentation. Visual Computing for Industry, Biomedicine, and Art, 3(1), 1-12.

DOI: 10.1186/S42492-020-00064-8.

[16]. Litjens, G., Kooi, T., Bejnordi, B. E., Setio, A. A. A., Ciompi, F., Ghafoorian, M., ... \& Sánchez, C. I. (2017). A survey on deep learning in medical image analysis. Medical image analysis, 42, 60-88.

https://doi.org/10.1016/j.media.2017.07.005

[17]. Fauzdar, P., \& Kumar, S. (2018). Computed Tomography Brain Images Semantic Segmentation. In Handbook of Research on Advanced Concepts in Real-Time Image and Video Processing (pp. 77-104). IGI Global. DOI: 10.4018/978-1-5225-2848-7.ch004

[18]. Dawod, A. Y., Abdullah, J., \& Alam, M. J. (2010, December). Adaptive skin color model for hand segmentation. In 2010 International Conference on Computer Applications and Industrial Electronics (pp. 486-489). IEEE.

DOI: 10.1109/ICCAIE.2010.5735129 
[19]. Thay, S., Aimmanee, P., Uyyanavara, B., \& Rukskul, P. (2018, February). Fast Hemorrhage Detection in Brain CT Scan Slices Using Projection Profile Based Decision Tree. In Proceedings of the 2018 International Conference on Intelligent Information Technology (pp. 18-21). DOI: $10.1145 / 3193063.3193073$

[20]. Patel, A., Schreuder, F. H., Klijn, C. J., Prokop, M., van Ginneken, B., Marquering, H. A., ... \& Manniesing, R. (2019). Intracerebral haemorrhage segmentation in non-contrast CT. Scientific reports, 9(1), 1-11.

DOI: $10.1038 / \mathrm{s} 41598-019-54491-6$

[21]. Li, L., Wei, M., Liu, B., Atchaneeyasakul, K., Zhou, F., Pan, Z., ... \& Scalzo, F. (2020). Deep learning for hemorrhagic lesion detection and segmentation on brain ct images. IEEE Journal of Biomedical and Health Informatics, 25(5), 1646-1659. DOI: 10.1109/JBHI.2020.3028243

[22]. Phan, A. C., Vo, V. Q., \& Phan, T. C. (2019). A Hounsfield value-based approach for automatic recognition of brain haemorrhage. Journal of Information and Telecommunication, 3(2), 196-209. https://doi.org/10.1080/24751839.2018.1547951

[23]. Malathi, M., \& Sinthia, P. (2019). Brain tumour segmentation using convolutional neural network with tensor flow. Asian Pacific journal of cancer prevention: APJCP, 20(7), 2095. DOI: 10.31557/APJCP.2019.20.7.2095

[24]. Ker, J., Singh, S. P., Bai, Y., Rao, J., Lim, T., \& Wang, L. (2019). Image thresholding improves 3dimensional convolutional neural network diagnosis of different acute brain hemorrhages on computed tomography scans. Sensors, 19(9), 2167. DOI: $10.3390 / \mathrm{s} 19092167$
[25]. Hssayeni, M. D., Croock, M. S., Salman, A. D., Alkhafaji, H. F., Yahya, Z. A., \& Ghoraani, B. (2020). Intracranial hemorrhage segmentation using a deep convolutional model. Data, 5(1), 14.

[26]. Chang, P. D., Kuoy, E., Grinband, J., Weinberg, B. D., Thompson, M., Homo, R., ... \& Chow, D. (2018). Hybrid 3D/2D convolutional neural network for hemorrhage evaluation on head CT. American Journal of Neuroradiology, 39(9), 1609-1616. DOI: 10.3174/ajnr.A5742

[27]. Ye, H., Gao, F., Yin, Y., Guo, D., Zhao, P., Lu, Y., ... \& Xia, J. (2019). Precise diagnosis of intracranial hemorrhage and subtypes using a three-dimensional joint convolutional and recurrent neural network. European radiology, 29(11), 6191-6201. DOI: $10.1007 / \mathrm{s} 00330-019-06163-2$

[28]. Mantas, J. (2020). Classification of intracranial hemorrhage subtypes using deep learning on CT scans. The Importance of Health Informatics in Public Health during a Pandemic, 272, 370. DOI: $10.3233 /$ SHTI200572

[29]. Burduja, M., Ionescu, R. T., \& Verga, N. (2020). Accurate and efficient intracranial hemorrhage detection and subtype classification in 3D CT scans with convolutional and long short-term memory neural networks. Sensors, 20(19), 5611. DOI: $10.3390 / \mathrm{s} 20195611$

[30]. Sage, A., \& Badura, P. (2020). Intracranial Hemorrhage Detection in Head CT Using DoubleBranch Convolutional Neural Network, Support Vector Machine, and Random Forest. Applied Sciences, 10(21), 7577. DOI: 10.3390/app10217577

[31]. Zhou, Z., Siddiquee, M. M. R., Tajbakhsh, N., \& Liang, J. (2018). Unet++: A nested u-net architecture for medical image segmentation. In Deep learning in medical image analysis and multimodal learning for clinical decision support (pp. 3-11). Springer, Cham. DOI: 10.1007/978-3-030-00889-5_1 